\title{
Prostate-Specific Antigen Screening According to Health Professional Counseling and Age in the United States
}

\author{
Ray M. Merrill $\mathbb{D}^{\mathrm{D}}$, Seth A. Otto $\mathbb{D}^{\mathrm{C}}$, and Eliza B. Hammond \\ Department of Public Health, College of Life Sciences, Brigham Young University, Provo, UT 84602, USA \\ Correspondence should be addressed to Ray M. Merrill; ray_merrill@byu.edu
}

Received 2 August 2021; Accepted 11 December 2021; Published 6 January 2022

Academic Editor: Ruhul Kuddus

Copyright ( 2022 Ray M. Merrill et al. This is an open access article distributed under the Creative Commons Attribution License, which permits unrestricted use, distribution, and reproduction in any medium, provided the original work is properly cited.

\begin{abstract}
Background. In 2018, the US Preventive Services Task Force recommended that PSA screening for prostate cancer involve men aged 55-69, based on a personal decision following consultation with a health professional. PSA screening in men aged 70 or older should only occur if symptoms exist. This study identifies the association between having a PSA test in the past two years and whether or not there was consultation with a health professional about the benefits and/or harms of PSA screening. Methods. Analyses were based on data involving men aged 40 years or older, who responded to PSA related questions in the 2018 BRFSS survey. Results. Approximately $32.0 \%$ (14.6\% for ages $40-54,41.7 \%$ for ages $55-69$, and $49.8 \%$ for ages 70 years and older) of respondents had a PSA test in the past two years. Approximately $81.7 \%$ of these men had talked with a health professional about the benefits and/or harms of PSA screening, with $42.4 \%$ having discussed the benefits and harms, $54.6 \%$ having discussed the benefits only, and 3.0\% having discussed the harms only. The odds of a PSA test in the past two years in men having talked with a health professional about the benefits and harms of the test versus no talk are 10.1 (95\% CI 9.3-10.8), in men who talked with a health professional about the benefits only versus no talk are 10.8 (95\% CI 10.0-11.6), and in men who talked with a health professional about the harms only versus no talk are 3.9 (95\% CI 2.9-5.1). Conclusion. PSA screening is most common in men aged 70 or older, which is counter to the US Preventive Task Force recommendation. Most men having a PSA test have talked with a health professional about the test, but the talks tended to focus on just the benefits of screening and not both potential benefits and harms.
\end{abstract}

\section{Introduction}

Prostate cancer is the most common nonskin cancer among men in the United States. In 2021, it accounted for an estimated $25.6 \%(248,530)$ of all cancer cases in males and $10.7 \%(34,130)$ of all cancer deaths in males [1]. There are over 3.2 million men currently alive in this country with a previous diagnosis of the disease [2]. The average lifetime risk of prostate cancer is $11.7 \%$ ( 1 in 8.5 ) for whites and $16.7 \%$ ( 1 in 6) for blacks, based on 2016-2018 Surveillance, Epidemiology, and End Results (SEER) data [3]. Average lifetime risk of dying from prostate cancer is $2.3 \%$ (1 in 43.5) for whites and 3.8\% (1 in 26.3) for blacks [3]. These results are consistent with prostate cancer being a slow-growing tumor such that men tend to die with the disease rather than from it [4-6].
Screening, diagnosis, and treatment for prostate cancer has been controversial in the past because of over diagnosis $[7,8]$. Prostate cancer screening may be associated with a decrease in prostate cancer death, though the evidence for this is conflicted [9]. Potential harmful effects of PSA screening include false-positive results and complications associated with resulting biopsy and treatment [9], such as adverse urinary symptoms and sexual dysfunction $[10,11]$. Nevertheless, in the past decade, important advances allow us to better characterize the likely progression of the disease following diagnosis and more effective treatment options are now available to patients $[7,12-16]$.

Previous reviews have summarized screening, diagnosis, and treatment options for prostate cancer [17-19]. Measurement of prostate-specific antigen (PSA) protein in the blood is the most common screening approach used for 
prostate cancer. In 2018, the US Preventive Services Task Force provided a recommendation statement regarding prostate cancer screening [20]. For men aged 55 to 69 years, PSA-based screening for prostate cancer should be an individual decision. The decision should follow a talk with their doctor about the clinical benefits (identifying high-risk early stage prostate cancer, which can be successfully treated) and harms (false positives, overdiagnosis, and treatment complications) of screening for prostate cancer. For men 70 years and older, routine PSA-based screening for prostate cancer is not recommended in the absence of symptoms. These recommendations apply to men of average or increased risk for prostate cancer who do not have symptoms of the disease and who have not been already diagnosed with prostate cancer.

The purpose of this study was to identify the prevalence of PSA testing in men aged 40 years or older, reasons for the test, and whether the respondents had ever talked with a health professional about the benefits and/or harms of PSA screening. The study assessed the association between having a PSA test in the past two years according to whether or not a health professional had talked with them about the benefits and/or harms of PSA screening or had recommended the test.

\section{Methods}

2.1. Data. The Behavioral Risk Factor Surveillance System (BRFSS) is a project wherein states in the United States (US) and participating US territories collaborate with the Center for Disease Control and Prevention (CDC). The BRFSS involves ongoing health-related telephone surveys designed to collect information on health-related risk behaviors, chronic health conditions, and access to preventive services. It is administered to noninstitutionalized adults in the US, aged 18 years and older [21]. The survey utilizes a random digit dialing technique on both cell phones and landlines to gather participants. Mean percent response rates for the 2018 BRFSS participating areas are $53.4 \%$ for landlines and $46.0 \%$ for cell phones (49.8\% combined) [22].

Analyses are restricted to 129,989 men aged 40 years or older who responded to whether or not they previously had a PSA test. Of this number, 3,806 had the test to monitor prostate cancer. These respondents were eliminated in our evaluation of PSA as a screening test, resulting in 126,183 respondents for evaluation.

2.2. Variables. Outcome variables include prevalence of a PSA test, reasons for the PSA test, whether or not a health professional had previously talked with the survey respondent about the benefits and/or harms of PSA screening, whether PSA screening had been recommended, and the percent of men who had a PSA test in the past two years. These outcome variables were determined from the following questions: "Have you ever had a PSA test?" "How long has it been since you had your PSA test?" "What are the main reasons you had this PSA test?" "Has a doctor, nurse, or other health professional ever talked with you about the benefits of the PSA test?" "Has a doctor, nurse, or other health professional ever talked with you about the harms of the PSA test?" "Has a doctor, nurse, or other health professional ever recommended that you have a PSA test?" These questions are listed in the 2018 BRFSS Codebook Report (https://www.cdc.gov/brfss/annual_data/2018/pdf/ codebook18_llcp-v2-508.pdf) [23].

Outcome variables were associated with and adjusted for race/ethnicity, age, marital status, education level, annual household income, BMI weight classification, whether they had smoked 100 or more cigarettes in the past, family history of prostate cancer, and health insurance. Race/ethnicity was classified as non-Hispanic white, non-Hispanic black, nonHispanic Asian, non-Hispanic American Indian/Alaskan Native, Hispanic, and non-Hispanic other race. Hereafter, we refer to these groups as white, black, Asian, American Indian/Alaskan Native, Hispanic, and other races. Questions upon which these variables are based are also included in the 2018 BRFSS Codebook Report [23].

2.3. Statistical Techniques. Frequencies, percentages, and odds ratios were calculated and reported from the sample survey data. Multiple logistic regression was used to estimate odds ratios adjusted for potential confounders. Odds ratios measured the association between having had PSA screening in the past two years and ever having had a talk with a health professional about the benefits and/or harms of PSA screening. Other variables were also associated with PSA screening in the past two years: race/ethnicity, age, marital status, education level, annual household income, smoking status, family history of prostate cancer, and health insurance. Person-level weights were applied to generate population estimates. Weighted percentages and odds ratios were reported in this paper. Statistical analyses were performed using SAS 9.4 (SAS Institute, Cary, NC, USA, 2012). SAS procedures used in the current study were SURVEYFREQ and SURVEYLOGISTIC. Graphs were created in Microsoft Excel (Microsoft Corporation, Issaquah, WA, USA, 2016).

\section{Results}

Selected aspects of PSA testing for men aged 40 years or older according to race/ethnicity appear in Table 1. Approximately $45.3 \%$ of men have ever had a PSA test, with the prevalence highest in whites and blacks and lowest in Asians and Hispanics. Having a PSA test as part of a routine exam is the most likely reason for the test, more so among Asians and Hispanics. Having a PSA test because of a prostate problem is also more common in Asians and Hispanics. Having a PSA test because of a family history of prostate cancer is more common in American Indians/Alaskan Natives. Finally, having a PSA test because of prostate cancer is more common in whites and blacks. Having ever talked with a health professional about the benefits of PSA screening, talked about the harms of PSA screening, been recommended PSA screening, or undergone a PSA screen in the past two years are each significantly more likely in whites 
TABLE 1: Prevalence of PSA testing, reasons for having the test, and talks with healthcare professionals about the test according to race/ ethnicity.

\begin{tabular}{|c|c|c|c|c|c|c|c|c|c|}
\hline & No. & $\%^{1}$ & White $\%^{1}$ & Black $\%{ }^{1}$ & Asian $\%^{1}$ & Am. Indian $\%^{1}$ & Hispanic $\%^{1}$ & Other $\%^{1}$ & $\begin{array}{c}\text { Rao-Scott } \\
\text { Chi-square } p \text {-value }\end{array}$ \\
\hline \multicolumn{10}{|l|}{ Ever had a PSA test } \\
\hline Yes & 67620 & 45.3 & 49.0 & 44.8 & 30.8 & 34.3 & 33.3 & 38.4 & $<0.0001$ \\
\hline No & 62369 & 54.7 & 51.0 & 55.2 & 69.2 & 65.7 & 66.7 & 61.6 & \\
\hline Missing & 7630 & & & & & & & & \\
\hline \multicolumn{10}{|c|}{ PSA test in the past two years } \\
\hline Yes & 45067 & 32.0 & 34.7 & 30.7 & 22.6 & 24.1 & 23.7 & 26.4 & $<0.0001$ \\
\hline No & 79203 & 68.0 & 65.3 & 69.3 & 77.4 & 75.9 & 76.3 & 73.6 & \\
\hline Missing & 1877 & & & & & & & & \\
\hline \multicolumn{10}{|c|}{ Reason for having a PSA test } \\
\hline Part of routine exam & 47165 & 71.4 & 71.6 & 67.7 & 75.3 & 70.4 & 73.8 & 69.3 & 0.0005 \\
\hline Prostate problem & 5174 & 7.6 & 7.2 & 8.0 & 9.0 & 6.9 & 9.1 & 7.5 & \\
\hline Family history of p.c. & 3869 & 5.8 & 6.2 & 5.7 & 4.4 & 7.3 & 4.2 & 4.2 & \\
\hline Prostate cancer & 3806 & 4.8 & 5.0 & 5.5 & 2.4 & 3.7 & 3.1 & 5.0 & \\
\hline Other reasons & 6725 & 10.4 & 10.1 & 13.1 & 9.0 & 11.7 & 9.8 & 14.1 & \\
\hline Missing & 881 & & & & & & & & \\
\hline \multicolumn{10}{|c|}{ Doctor, nurse, or other health professional ever talked with you about the benefits of the PSA test ${ }^{2}$} \\
\hline Yes & 60594 & 43.7 & 47.10 & 45.76 & 34.19 & 34.31 & 29.81 & 37.52 & $<0.0001$ \\
\hline No & 62498 & 56.3 & 52.90 & 54.24 & 65.81 & 65.70 & 70.19 & 62.48 & \\
\hline Missing & 3091 & & & & & & & & \\
\hline \multicolumn{10}{|c|}{ Doctor, nurse, or other health professional ever talked with you about the harms of the PSA screennig ${ }^{2}$} \\
\hline Yes & 27854 & 20.0 & 20.4 & 25.2 & 16.0 & 17.8 & 16.1 & 17.2 & $<0.0001$ \\
\hline No & 94709 & 78.0 & 79.6 & 74.8 & 84.0 & 82.2 & 83.9 & 82.8 & \\
\hline Missing & 3620 & & & & & & & & \\
\hline \multicolumn{10}{|c|}{ Doctor, nurse, or other health professionals ever recommend that you have a PSA screening ${ }^{2}$} \\
\hline Yes & 56256 & 40.4 & 43.4 & 41.5 & 29.6 & 29.7 & 29.4 & 33.6 & $<0.0001$ \\
\hline No & 67050 & 59.6 & 56.6 & 58.5 & 70.4 & 70.3 & 70.6 & 66.4 & \\
\hline Missing & 2677 & & & & & & & & \\
\hline
\end{tabular}

and blacks. Talking about the benefits is approximately twice as common as talking about the harms, similarly across the racial/ethnic groups.

The percent of respondents who indicated that a health professional had ever talked with them about benefits and/or harms of PSA screening is shown according to the level of selected variables in Figure 1. A pattern of greater talk about only the benefits of PSA screening appears across the levels of each of the variables in the figure. In general, ever having talked about PSA screening with a health professional increased with age $(24.5 \%$ in ages $40-54,53.3 \%$ in ages $55-69$, and $59.6 \%$ in ages 70 or older). Ever having talked with a health professional about both the benefits and harms of PSA screening occurred in $18.1 \%$ (10.9\% in ages $40-54$, $22.6 \%$ in ages $55-69$, and $24.0 \%$ in ages 70 or older) of men, about the benefits only in $23.3 \%$ (12.5\% in ages $40-54,29.3 \%$ in ages $55-69$, and $33.8 \%$ in ages 70 or older) of men, and about the harms only in $1.3 \%$ (1.0\% in ages $40-54,1.3 \%$ in ages $55-69$, and $1.8 \%$ in ages 70 or older) of men. Thus, it is most common for a health professional to talk about the benefits only, followed by both the benefits and harms and then the harms only. The ratio of talk about just benefits versus talk about both benefits and harms increased across the three age groups, from $14.7 \%$ greater to $29.6 \%$ greater and to $40.8 \%$ greater.
The percent of men who had a PSA test in the past two years according to whether they had a previous recommendation for the test from a health professional is shown in Figure 2. In each age group, the percent having a PSA test in the past two years was strongly influenced by whether it was recommended by a health professional. Specifically, if PSA screening was ever recommended versus otherwise, having a PSA test in the past two years was 21.5 times greater in ages 40-49, 9.6 times greater in ages 50-59, 5.6 times greater in ages 60-69, 4.4 times greater in ages 70-79, and 4.6 times greater in ages 80 or older. The percent of respondents ever recommended PSA screening was $13.8 \%$ in ages $40-49$, $38.2 \%$ in ages $50-59,55.2 \%$ in ages $60-69,62.0 \%$ in ages $70-79$, and $52.6 \%$ in ages 80 or older. Overall, when a doctor, nurse, or other health professionals ever recommended PSA screening, $68.8 \%$ had a PSA test in the past two years, compared with $7.5 \%$ otherwise.

PSA screening in the past two years was significantly greater among men who had a health professional ever talk with them about the benefits and/or harms of PSA screening (Table 2). Although talking about the benefits is most strongly associated with PSA screening in the past two years, talking about the harms only was also positively associated with PSA screening in the past two years. Without adjustment for potential confounders, whites were significantly 


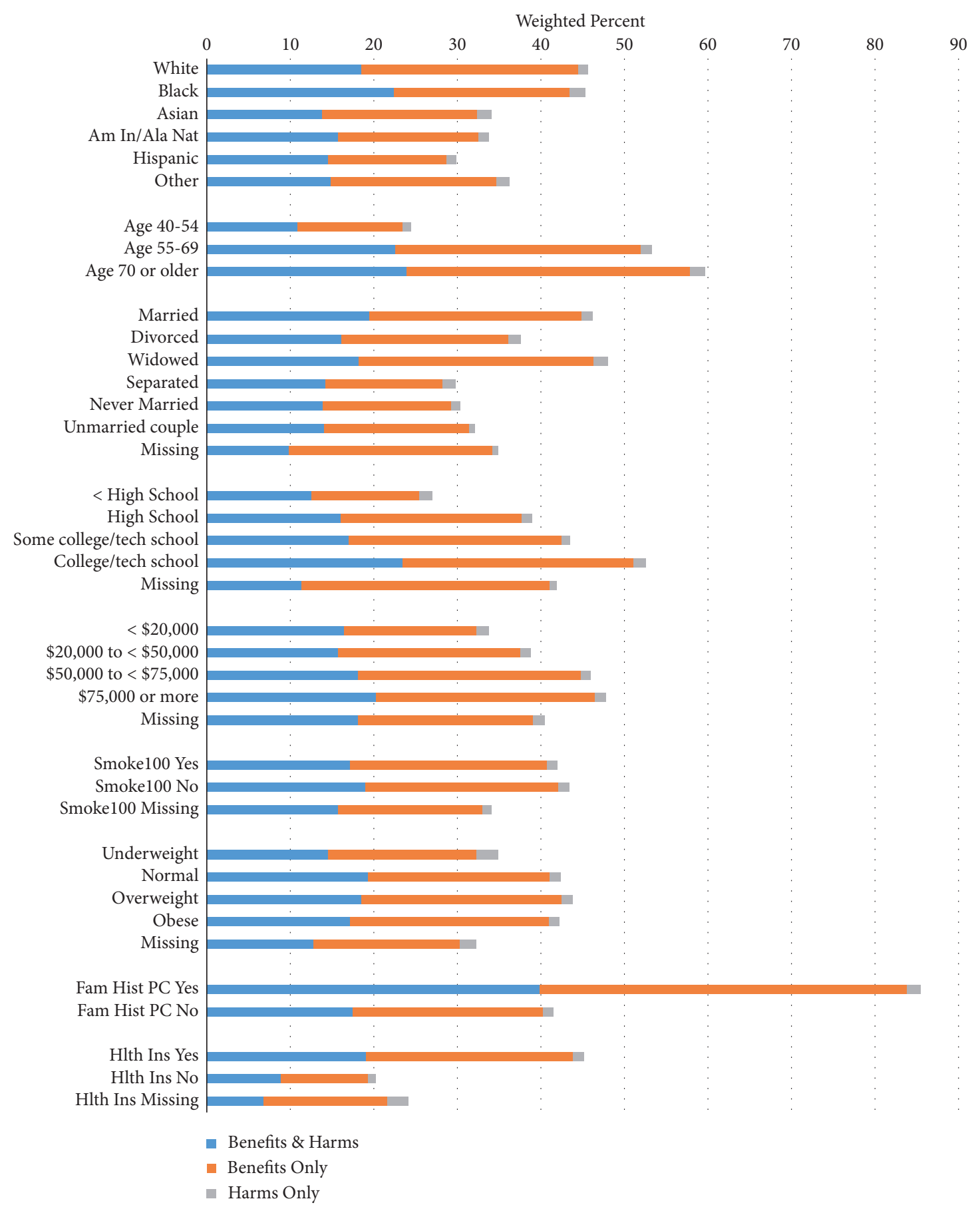

FIGURE 1: Health professional ever talked with the survey respondent about benefits and/or harms of PSA screening according to selected variables.

more likely to receive PSA screening in the past two years. However, after adjusting for education, annual household income, and other variables, blacks and Hispanics (versus whites) were significantly more likely to have PSA screening in the past two years. PSA screening in the past two years was also positively associated with age and was more common among men who were married, had higher education, had higher annual household income, had not smoked at least 100 cigarettes in their lifetime, were overweight or obese, had a family history of prostate cancer, and were currently insured.
Prevalence of having had a PSA test in the past two years among men aged 40 years and older is $32.0 \%$ (14.6\% for ages $40-54,41.7 \%$ for ages $55-69$, and $49.8 \%$ for ages 70 years and older). Prevalence of ever having discussed the benefits and/ or harms of a PSA test with a health professional for these men who received the test was $81.7 \%$ (76.6\% for ages $40-54$, $83.3 \%$ for ages $55-69$, and $82.3 \%$ for ages 70 years and older).

PSA screening in the past two years according to age and race/ethnicity appears in Figure 3. PSA screening is most common in the age group 70-79 years, except for Hispanics, 


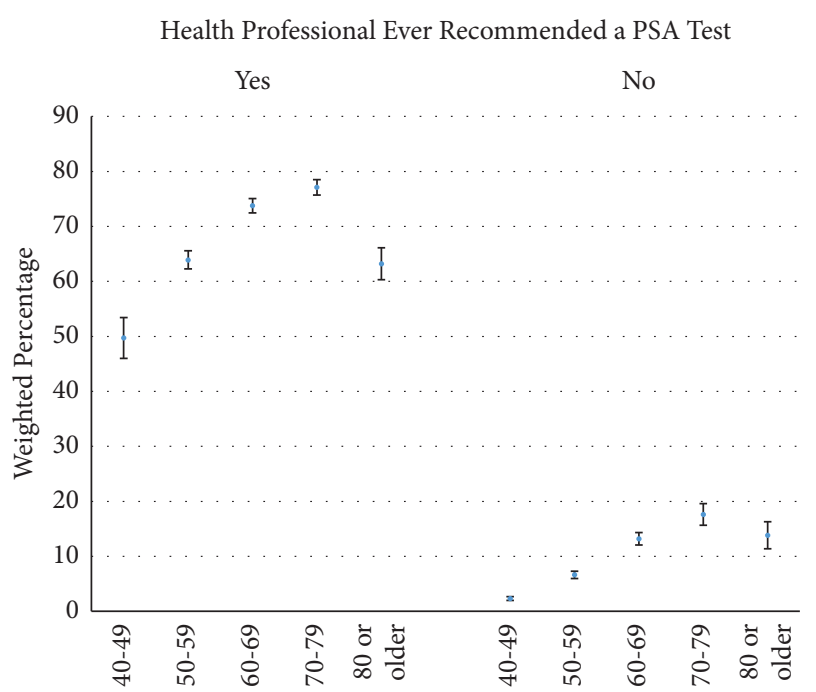

FIgURe 2: PSA test in the past two years according to a previous recommendation for the test from a health professional and age.

where it is the greatest in the age group 80 or older. In the age groups 40-49 and 50-59, PSA screening in the past two years is most common among blacks and least common among Asians. In the age groups 60-69 and 70-79, PSA screening in the past two years is most common among whites and blacks and least common among American Indians/Alaskan Natives and Hispanics. In the age group 80 years or older, PSA screening in the past two years decreases, except for among Hispanics.

\section{Discussion}

This study identified the prevalence of a health professional ever having talked with the survey respondents about the benefits and/or harms of PSA screening, according to age and other variables. It also investigated how this talk and recommendation for PSA screening is associated with PSA screening in the past two years. Further, we presented the level PSA screening in the past two years according to age and other variables.

Previous research indicates that PSA screening has the potential of overdiagnosing patients and increasing prostate cancer incidence among men more likely to die from other causes before prostate cancer symptoms manifest themselves [4-8, 24]. Potential harmful effects of PSA screening (e.g., false-positive results and complications associated with resulting biopsy and treatment $[9-11,17,25])$ led the US Preventive Services Task Force to recommend that for men aged 55 to 69, PSA-based screening for prostate cancer should be an individual decision, following a talk with their doctor about the benefits and harms of screening for prostate cancer [20]. For men 70 years and older, routine PSA-based screening for prostate cancer should not be done for men who do not have symptoms of the disease [20].

It is recommended that men have the chance to discuss the benefits and harms of PSA screening with their primary care provider as they make decisions about screening $[25,26]$. Nevertheless, only $42.7 \%(24.4 \%$ in ages $40-54$,
$53.2 \%$ in ages $55-69$, and $59.6 \%$ in ages 70 or older) of the men surveyed reported having previously had a talk with a health professional about the benefits and/or harms of PSA screening. Further, the talks tended to focus on just the benefits of PSA screening (i.e., 23.3\%, 12.5\% in ages $40-54$, $29.3 \%$ in ages $55-69$, and $33.8 \%$ in ages 70 or older) and not on both the benefits and harms of PSA screening (i.e., 18.1\%, $10.9 \%$ in ages $40-54,22.6 \%$ in ages $55-69$, and $24.0 \%$ in ages 70 or older). Talking about just the harms of PSA screening occurred less than $2 \%$ of the time. Thus, in most of the cases where a health professional talked with the participants about PSA screening, the talk was not a balanced discussion and the ability of the man to make an informed decision was comprised.

PSA screening in the past two years peaked in ages 70-79 and decreased thereafter. A drop in the oldest ages may be motivated by recommendations discouraging PSA screening in older ages $[20,25]$. These recommendations are influenced by prostate cancer being a slow growing tumor and five-year survival improving with older age $[4-7,27,28]$. Nevertheless, the high level of PSA screening occurring in the ages $70-79$ and 80 or older is in contrast to what is recommended by the US Preventive Services Task Force.

Recommendation for PSA screening significantly positively associated with PSA screening in the past two years. The impact of recommendation on PSA screening was most pronounced in the younger age groups. Recommendation for PSA screening in a previous study showed that PSA testing is positively associated with a physician's direct communication about prostate cancer and encouragement to be screened [26].

In the remainder of this discussion, we will talk about the results involving race/ethnicity and other selected variables. Whites and blacks were more likely to have ever had a talk with a health professional about the benefits and/or harms of PSA screening. Whites and blacks were also more likely to have a health professional ever recommend PSA screening. These results may be because blacks have significantly higher rates of prostate cancer than the other racial/ethnic groups, followed by whites [29]. In addition, whites and blacks have the highest level of a family history of prostate cancer and whites and Asians have the highest levels of marriage, education, income, and health insurance (data not shown), each of which has been associated with increased levels of prostate cancer screening [30-32].

The significantly higher level of PSA screening in the past two years among whites and blacks is consistent with their having a higher level of health professionals talk with them about the benefits and/or harms of PSA testing, as well as recommending the test. However, blacks and Hispanics had the lowest levels of education, income, and insurance among the racial/ethnic groups (data not shown), which are buoying factors for higher PSA screening.

In the adjusted model (Table 2), blacks and Hispanics, compared with whites, experienced higher PSA screening in the past two years.

PSA screening in the past two years shows an increase until peaking in the age group 70-79 and then decreases. This is true for all racial/ethnic groups except for Hispanics, 
TABLE 2: Odds of receiving a PSA test in the past two years according to whether or not a health professional had ever talked with the respondent about the benefits and/or harms of PSA testing.

PSA in the past two years unadjusted PSA in the past two years adjusted for the other variables in the table

No. Col\% Row \% Odds ratio 95\% LCL 95\% UCL $\quad$ Pr $>|t|$ Odds ratio 95\% LCL 95\% UCL $\operatorname{Pr}>|t|$

\begin{tabular}{|c|c|c|c|c|c|c|c|c|c|c|c|}
\hline \multicolumn{12}{|c|}{ Talk about PSA with health professional } \\
\hline Benefits \& harms & 27408 & 18.4 & 61.2 & 13.93 & 12.98 & 14.95 & $<0.0001$ & 10.07 & 9.34 & 10.85 & $<0.0001$ \\
\hline Benefits only & 34532 & 23.9 & 63.3 & 15.24 & 14.20 & 16.35 & & 10.76 & 9.99 & 11.58 & \\
\hline Harms only & 1598 & 1.3 & 36.0 & 4.98 & 3.91 & 6.35 & & 3.87 & 2.94 & 5.10 & \\
\hline Neither & 66451 & 56.4 & 10.2 & 1 & & & & 1 & & & \\
\hline \multicolumn{12}{|l|}{ Race/ethnicity } \\
\hline White & 100435 & 68.6 & 34.7 & 1 & & & $<0.0001$ & 1 & & & $<0.0001$ \\
\hline Black & 8746 & 10.4 & 30.7 & 0.83 & 0.77 & 0.91 & & 1.14 & 1.02 & 1.27 & \\
\hline Asian & 2520 & 4.4 & 22.6 & 0.55 & 0.44 & 0.68 & & 0.68 & 0.54 & 0.85 & \\
\hline Am. Indian/Alaskan Nat & 2359 & 1.0 & 24.1 & 0.60 & 0.49 & 0.73 & & 0.89 & 0.70 & 1.14 & \\
\hline Hispanic & 8242 & 13.9 & 23.7 & 0.58 & 0.52 & 0.65 & & 1.26 & 1.10 & 1.45 & \\
\hline Other & 3881 & 1.8 & 26.4 & 0.68 & 0.59 & 0.78 & & 0.97 & 0.81 & 1.16 & \\
\hline \multicolumn{12}{|l|}{ Age groups } \\
\hline $40-54$ & 37152 & 41.3 & 14.6 & 1 & & & $<0.0001$ & 1 & & & $<0.0001$ \\
\hline $55-69$ & 54197 & 38.8 & 41.7 & 4.19 & 3.92 & 4.47 & & 2.86 & 2.65 & 3.09 & \\
\hline 70 or older & 34834 & 20.0 & 49.8 & 5.80 & 5.39 & 6.25 & & 3.84 & 3.51 & 4.21 & \\
\hline \multicolumn{12}{|l|}{ Marital status } \\
\hline Married & 78406 & 65.0 & 35.5 & 1 & & & $<0.0001$ & 1 & & & $<0.0001$ \\
\hline Divorced & 19436 & 13.8 & 27.0 & 0.67 & 0.62 & 0.72 & & 0.87 & 0.80 & 0.96 & \\
\hline Widowed & 9739 & 5.5 & 37.1 & 1.07 & 0.96 & 1.19 & & 0.81 & 0.72 & 0.93 & \\
\hline Separated & 2685 & 2.5 & 20.0 & 0.45 & 0.38 & 0.55 & & 0.79 & 0.64 & 0.97 & \\
\hline Never married & 12751 & 10.3 & 19.6 & 0.44 & 0.41 & 0.49 & & 0.73 & 0.65 & 0.82 & \\
\hline Unmarried couple & 2720 & 2.6 & 21.8 & 0.51 & 0.42 & 0.60 & & 0.79 & 0.64 & 0.98 & \\
\hline Missing & 446 & 0.3 & 24.8 & 0.60 & 0.42 & 0.86 & & 0.80 & 0.52 & 1.22 & \\
\hline \multicolumn{12}{|l|}{ Education level } \\
\hline$<$ High school & 9678 & 14.2 & 19.9 & 0.61 & 0.55 & 0.68 & $<0.0001$ & 0.82 & 0.72 & 0.94 & $<0.0001$ \\
\hline High school & 33847 & 27.1 & 29.0 & 1 & & & & 1 & & & \\
\hline Some college/tech school & 31726 & 28.5 & 32.9 & 1.20 & 1.12 & 1.29 & & 1.04 & 0.96 & 1.13 & \\
\hline College/tech school & 50686 & 30.0 & 39.7 & 1.61 & 1.52 & 1.71 & & 1.18 & 1.09 & 1.27 & \\
\hline Missing & 246 & 0.2 & 28.5 & 0.98 & 0.51 & 1.86 & & 0.71 & 0.40 & 1.25 & \\
\hline \multicolumn{12}{|l|}{ Annual household income } \\
\hline$<\$ 20,000$ & 15098 & 12.5 & 22.7 & 0.72 & 0.65 & 0.79 & $<0.0001$ & 0.88 & 0.79 & 0.99 & $<0.0001$ \\
\hline$\$ 20,000$ to $<\$ 50,000$ & 33607 & 25.6 & 29.0 & 1 & & & & 1 & & & \\
\hline$\$ 50,000$ to $<\$ 75,000$ & 18509 & 13.8 & 34.5 & 1.29 & 1.19 & 1.40 & & 1.10 & 0.99 & 1.22 & \\
\hline$\$ 75,000$ or more & 44506 & 36.7 & 35.9 & 1.37 & 1.29 & 1.46 & & 1.21 & 1.10 & 1.32 & \\
\hline Missing & 14463 & 11.5 & 33.4 & 1.23 & 1.12 & 1.35 & & 1.27 & 1.13 & 1.42 & \\
\hline \multicolumn{12}{|c|}{ Have smoked at least 100 cigarettes in lifetime } \\
\hline Yes & 64850 & 50.9 & 31.4 & 1 & & & 0.0430 & 1 & & & $<0.0001$ \\
\hline No & 60646 & 48.5 & 32.7 & 1.06 & 1.01 & 1.12 & & 1.08 & 1.02 & 1.15 & \\
\hline Missing & 687 & 0.6 & 29.9 & 0.931 & 0.70 & 1.24 & & 1.26 & 0.88 & 1.80 & \\
\hline \multicolumn{12}{|l|}{ Body mass index category } \\
\hline Underweight & 954 & 0.7 & 23.7 & 0.70 & 0.55 & 0.90 & $<0.0001$ & 0.89 & 0.67 & 1.17 & $<0.0001$ \\
\hline Normal weight & 27283 & 21.0 & 30.6 & 1 & & & & 1 & & & \\
\hline Overweight & 53374 & 42.4 & 33.1 & 1.12 & 1.05 & 1.20 & & 1.14 & 1.05 & 1.24 & \\
\hline Obese & 42285 & 33.7 & 32.5 & 1.09 & 1.02 & 1.17 & & 1.25 & 1.14 & 1.36 & \\
\hline Missing & 2287 & 2.2 & 19.8 & 0.56 & 0.47 & 0.68 & & 0.77 & 0.59 & 1.01 & \\
\hline \multicolumn{12}{|c|}{ Family history of prostate cancer } \\
\hline Yes & 3869 & 2.7 & 74.0 & 6.39 & 5.46 & 7.48 & $<0.0001$ & 3.37 & 2.67 & 4.24 & $<0.0001$ \\
\hline No & 122314 & 97.3 & 26.0 & 1 & & & & 1 & & & \\
\hline \multicolumn{12}{|c|}{ Current health insurance status } \\
\hline Yes & 116996 & 90.0 & 34.2 & 3.89 & 3.34 & 4.54 & $<0.0001$ & 1.74 & 1.47 & 2.05 & $<0.0001$ \\
\hline No & 8864 & 9.7 & 11.8 & 1.00 & & & & 1 & & & \\
\hline Missing & 323 & 0.3 & 22.2 & 2.13 & 1.25 & 3.64 & & 2.01 & 1.11 & 3.63 & \\
\hline
\end{tabular}

LCL: lower confidence level; UCL: upper confidence level. The results in this table apply to male respondents aged 40 years or older. Those who had a PSA test to monitor prostate cancer are not included. Four categories of BMI: underweight BMI $<18.5$, normal weight $18.5 \leq \mathrm{BMI}<25$, overweight $25 \leq \mathrm{BMI}<30$, and obese $30 \leq$ BMI. Percentages and odds ratios (with corresponding confidence intervals) are weighted in order to get a representative sample of the US population. Confidence intervals that do not overlap 1 indicate statistical significance at the 0.05 level. 


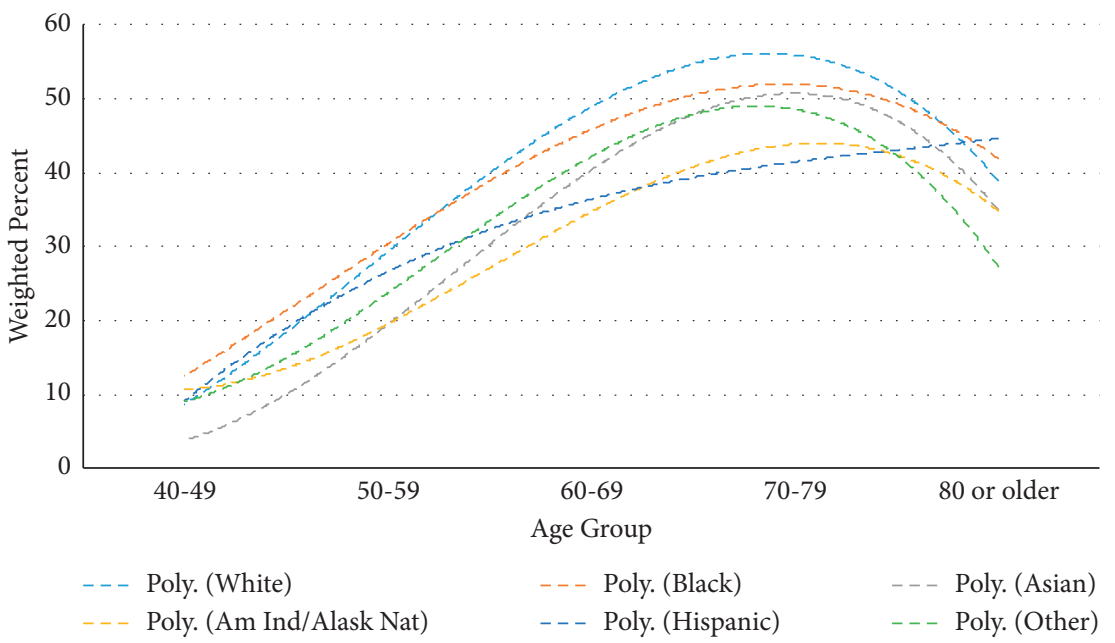

FIgURE 3: PSA test in the past two years according to age and race/ethnicity (data fit using third-order polynomial models).

where the rate is greatest in the age group 80 or older. According to the guidelines [20], the decrease in PSA screening should have peaked at least a decade earlier for each of the racial/ethnic groups. However, the rate of increase with increasing age groups is decreasing for each racial/ethnic group through ages 70-79.

In the adjusted model (Table 2), married men had significantly higher levels of PSA testing in the past two years. This is consistent with previous literature showing that men who have a spouse or partner are more likely to be screened for prostate cancer [33-35]. This is likely because they have an independent party involved and invested in their health.

Higher education and higher annual household income were both associated with significantly greater PSA screening in the past two years. These two variables are usually connected to the broader term-socioeconomic status-a key determinant of health [36]. As such, higher education and income have been closely linked to greater PSA screening [37, 38]. Higher education and income are associated with increased knowledge and access to quality healthcare, which allows a patient to better understand and react to their situation. Thus, not only are these patients likely to be more informed about prostate cancer and PSA screening overall, but also they are better equipped to obtain the PSA test because of their greater financial resources.

Men who smoked at least 100 cigarettes in their lifetime were significantly less likely to have had a PSA test in the past two years. Other research has shown that current smokers are significantly less likely to undergo PSA screening $[39,40]$. In the current study, lower PSA screening among smokers persisted after adjusting for several other variables. Smoking may represent a host of poor health behaviors, such as compromised consumption of healthy food, inadequate exercise, and risky sexual behavior [41, 42].

The analysis showed that overweight and obese individuals were more likely to have had a PSA test in the past two years. These results became more pronounced in the adjusted model. Previous research has likewise shown that overweight and obesity are associated with greater PSA testing [43]. This may be due to existing health concerns or comorbid factors that cause these individuals to be more conscientious of arising health issues. Additionally, it is possible that these individuals, because they are less healthy, are more likely to frequently check-in with their doctor [44].

Individuals with a family history of prostate cancer were significantly more likely to have a PSA test in the past two years. This group was far more likely than any other group (i.e., $85.4 \%$ ) to have ever had a talk with a health professional about the benefits and/or harms of PSA screening. Approximately $39.9 \%$ had ever had a talk with a health professional about the benefits and harms, $43.9 \%$ had ever had a talk with a health professional about the benefits only, and $1.6 \%$ had ever had a talk with a health professional about the harms only. Among the variables considered in this study, ever having talked with a health professional about the benefits and/or harms of PSA screening and a family history of prostate cancer were the two leading factors associated with a PSA test in the past two years.

Those with some form of health insurance (prepaid plans or government plans) were also more likely to undergo PSA screening in the past two years. Other research has shown a direct association between health insurance and cancer testing, specifically involving cervical cancer, colon cancer, and mammography [45].

Some limitations exist in this study. First, response rates average $49.8 \%$. Low response rates may have resulted in selection bias. Research has shown that telephone-based survey response rates have recently decreased and are lower than in-person response rates. However, similar research findings have indicated that applying weight to demographic variables of participants generally allows for accurate measurements [21]. Second, this is a cross-sectional survey where poor recall may be an issue. Third, interpretation is limited to discussing associations rather than cause-effect relationships.

\section{Conclusion}

5.1. PSA Screening. Although the US Preventive Services Task Force general guidelines recommend that men begin PSA screening at age 55 and stop at age 70 , unless symptoms 
are present, a large proportion of men in the age range 40-54 and 70 and older undergo PSA screening. In our US sample, PSA screening in the past two years is greatest among men ages 70-79 years. The current study shows that talking with a health professional about PSA screening or receiving a recommendation for screening has a large impact on the decision to be screened. Hence, health professionals should be more sensitive to the age guidelines for screening as they counsel patients.

5.2. Informed Decision-Making. Undergoing PSA screening for prostate cancer should be an individual decision, but in conjunction with consultation with a health professional about the potential benefits and harms of screening. In our sample of men who were PSA screened in the past two years, approximately $81.7 \%$ (76.6\% in ages $40-54,83.3 \%$ for ages $55-69$, and $82.3 \%$ for ages 70 years and older) had previously talked with a health professional about the benefits and/or harms of PSA screening. Hence, there is a need for many men undergoing PSA testing who have not first talked with a health professional about both the potential benefits and harms of the test to do so.

When talks occurred with a health professional about PSA screening, $42.4 \%$ involved discussion about the benefits and harms, $54.6 \%$ involved discussion about the benefits only, and $3.0 \%$ involved discussion about the harms only. Thus, health professionals should do a better job providing a balanced discussion with their patients of the benefits and harms of PSA screening.

5.3. Factors Associated with PSA Screening and Consultation. Other factors associated with higher levels of PSA screening are being white, being older in age, being married, being a college or technical school graduate, having an annual household income of $\$ 75,000$ or more, not having smoked at least 100 cigarettes in their lifetime, being overweight or obese, having a family history of prostate cancer, and having health insurance. Having a health professional talk with a patient about the potential benefits and/or harms of PSA screening or having a family history of prostate cancer had the greatest positive association with PSA screening.

Health professionals are most likely to discuss benefits and/or harms of PSA screening with whites and blacks and least likely with Hispanics. They are also more likely to recommend PSA screening for whites and blacks. These differences in discussion and recommendation are likely explained, at least in part, by differences among the racial/ ethnic groups in levels of marriage, education, annual household income, and insurance.

\section{Abbreviations}

BRFSS: Behavior Risk Factor Surveillance System

CDC: Centers for Disease Control and Prevention

PSA: Prostates Specific Antigen

SAS: Statistical Analysis System

SEER: Surveillance, Epidemiology, and End Results

US: United States.

\section{Data Availability}

The data used to support the findings of this study are available from the corresponding author upon request.

\section{Ethical Approval}

The data used in this analysis were deemed exempt by the authors' Institutional Review Board.

\section{Conflicts of Interest}

None of the contributing authors have any conflicts of interest.

\section{References}

[1] American Cancer Society (ACS), Cancer Facts \& Figures 2021, American Cancer Society, Atlanta, GA, USA, https://www. cancer.org/content/dam/cancer-org/research/cancer-factsand-statistics/annual-cancer-facts-and-figures/2021/cancerfacts-and-figures-2021.pdf.

[2] Surveillance Epidemiology and End Results Program, Cancer Stat Facts: Prostate Cancer 2018, National Cancer Institute (NCI), Bethesda, ML, USA, https://seer.cancer.gov/statfacts/ html/prost.html.

[3] N. Howlader, A. M. Noone, M. Krapcho et al., Eds., SEER Cancer Statistics Review, 1975-2018, National Cancer Institute, Bethesda, MD, USA, 2021, https://seer.cancer.gov/csr/ 1975_2018/ based on November 2020 SEER data submission, posted to the SEER web site.

[4] T. J. Daskivich, K.-H. Fan, T. Koyama et al., "Effect of age, tumor risk, and comorbidity on competing risks for survival in a U.S. Population-based cohort of men with prostate cancer," Annals of Internal Medicine, vol. 158, no. 10, pp. 709-717, 2013.

[5] J. J. Fenton, M. S. Weyrich, S. Durbin, Y. Liu, H. Bang, and J. Melnikow, Prostate-Specific Antigen-Based Screening for Prostate Cancer: A Systematic Evidence Review for the US Preventive Services Task Force, Agency for Healthcare Research and Quality, Rockville, MD, USA, AHRQ Publication No. 17-05229-EF-1, 2018.

[6] T. J. Wilt, M. K. Brawer, K. M. Jones et al., "Radical prostatectomy versus observation for localized prostate cancer," New England Journal of Medicine, vol. 367, no. 3, pp. 203-213, 2012.

[7] A. Bill-Axelson, L. Holmberg, H. Garmo et al., "Radical prostatectomy or watchful waiting in early prostate cancer," New England Journal of Medicine, vol. 370, no. 10, pp. 932-942, 2014.

[8] F. H. Schröder, J. Hugosson, M. J. Roobol et al., "ERSPC Investigators, Screening and prostate-cancer mortality in a randomized European study," New England Journal of Medicine, vol. 360, pp. 1320-1328, 2009.

[9] J. H. Hayes and M. J. Barry, "Screening for prostate cancer with the prostate-specific antigen test," JAMA, vol. 311, no. 11, pp. 1143-1149, 2014.

[10] D. A. Barocas, J. Alvarez, M. J. Resnick et al., “Association between radiation therapy, surgery, or observation for localized prostate cancer and patient-reported outcomes after 3 years," JAMA, vol. 317, no. 11, pp. 1126-1140, 2017.

[11] R. C. Chen, R. Basak, A.-M. Meyer et al., "Association between choice of radical prostatectomy, external beam radiotherapy, brachytherapy, or active surveillance and patient-reported 
quality of life among men with localized prostate cancer," JAMA, vol. 317, no. 11, pp. 1141-1150, 2017.

[12] A. Bill-Axelson, L. Holmberg, H. Garmo et al., "Radical prostatectomy or watchful waiting in prostate cancer-29-year follow-up," New England Journal of Medicine, vol. 379, no. 24, pp. 2319-2329, 2018.

[13] Z. Yang, Z. R. McCaw, and G. Yin, "Correspondence: radical surgery or watchful waiting in prostate cancer," New England Journal of Medicine, vol. 380, pp. 1083-1084, 2019.

[14] B. W. Goy, R. Burchette, M. S. Soper, T. Chang, and H. A. Cosmatos, "Ten-year treatment outcomes of radical prostatectomy vs external beam radiation therapy vs brachytherapy for 1503 patients with intermediate-risk prostate cancer," Urology, vol. 136, pp. 180-189, 2020.

[15] A. V. D’Amico, R. Whittington, S. B. Malkowicz et al., "Biochemical outcome after radical prostatectomy, external beam radiation therapy, or interstitial radiation therapy for clinically localized prostate cancer," JAMA, vol. 280, no. 11 , pp. 969-974, 1998.

[16] M. T. Schweizer and E. S. Antonarakis, "Chemotherapy and its evolving role in the management of advanced prostate cancer," Asian Journal of Andrology, vol. 16, pp. 334-340, 2014.

[17] J. B. Aragon-China, V. Lohiva, and G. Sonpovde, "Role of chemotherapy and mechanisms of resistance to chemotherapy in metastatic castration-resistant prostate cancer," The Oncologist, vol. 10, pp. 57-66, 2016.

[18] M. J. Barry and J. H. Hayes, "Evaluating an elevated screening PSA test," JAMA, vol. 314, no. 19, pp. 2073-2074, 2015.

[19] M. S. Litwin and H.-J. Tan, "The diagnosis and treatment of prostate cancer," JAMA, vol. 317, no. 24, pp. 2532-2542, 2017.

[20] Prostate cancer: screening, U.S. Preventive Services Task Force, 2021, https://www.uspreventiveservicestaskforce.org/ uspstf/recommendation/prostate-cancer-screening.

[21] Behavior Risk Factor Surveillance System: Overview, Centers for Disease Control and Prevention, 2021, https://www.cdc. gov/brfss/annual_data/2016/pdf/overview_2016.pdf.

[22] Behavioral Risk Factor Surveillance System: 2018 Summary Data Quality Report, Centers for Disease Control and Prevention, 2021, https://stacks.cdc.gov/view/cdc/81722.

[23] Behavioral Risk Factor Surveillance System, LLCP 2018 Codebook Report, Centers for Disease Control and Prevention, Atlanta, GA, USA.

[24] R. M. Merrill, A. L. Potosky, and E. J. Feuer, "Changing trends in U.S. prostate cancer incidence rates," JNCI Journal of the National Cancer Institute, vol. 88, no. 22, pp. 1683-1685, 1996.

[25] S. H. Woolf and A. Krist, "Shared decision making for prostate cancer screening," Archives of Internal Medicine, vol. 169, no. 17, pp. 1557-1559, 2009.

[26] V. D. Woods, S. B. Montgomery, R. P. Herring, R. W. Gardner, and D. Stokols, "Social ecological predictors of prostate-specific antigen blood test and digital rectal examination in black American men," Journal of the National Medical Association, vol. 98, pp. 492-504, 2006.

[27] R. M. Merrill and J. S. Bird, "Effect of young age on prostate cancer survival: a population-based assessment (United States)," Cancer Causes \& Control, vol. 13, no. 5, pp. 435-443, 2002.

[28] C. A. Salinas, A. Tsodikov, M. Ishak-Howard, and K. A. Cooney, "Prostate cancer in young men: an important clinical entity," Nature Reviews Urology, vol. 11, no. 6, pp. 317-323, 2014.

[29] Cancer Stat Facts: Prostate Cancer, 2021, https://seer.cancer. gov/statfacts/html/prost.html.

[30] J. Li, G. Zhao, L. A. Pollack, J. L. Smith, and D. A. Joseph, "Use of the prostate-specific antigen test among men aged 75 years or older in the United States: 2006 Behavioral Risk Factor Surveillance System," Preventing Chronic Disease, vol. 7, no. 4, p. A84, 2010.

[31] M. E. McDowell, S. Occhipinti, R. A. Gardiner, and S. K. Chambers, "Patterns of prostate-specific antigen (PSA) testing in Australian men: the influence of family history," BJU International, vol. 109, pp. 64-70, 2012.

[32] M. E. Ogunsanya, S. Jiang, A. V. Thach, B. A. Bamgbade, and C. M. Brown, "Predictors of prostate cancer screening using andersen's behavioral model of health services use," Urologic Oncology, vol. 34, p. 529, 2016.

[33] A. A. Kotwal, P. Schumm, S. G. Mohile, and W. Dale, "The influence of stress, depression, and anxiety on PSA screening rates in a nationally representative sample," Medical Care, vol. 50, no. 12, pp. 1037-1044, 2012.

[34] E. M. Flahavan, F. J. Drummond, K. Bennett, T. I. Barron, and L. Sharp, "Prostate specific antigen testing is associated with men's psychological and physical health and their healthcare utilisation in a nationally representative sample: a crosssectional study," BMC Family Practice, vol. 15, no. 1, p. 121, 2014.

[35] T. J. Littlejohns, R. C. Travis, T. J. Key, and N. E. Allen, "Lifestyle factors and prostate-specific antigen (PSA) testing in UK Biobank: implications for epidemiological research," Cancer Epidemiology, vol. 45, pp. 40-46, 2016.

[36] P. Braveman and L. Gottlieb, "The social determinants of health: it's time to consider the causes of the causes," Public Health Reports, vol. 129, no. 1_suppl2, pp. 19-31, 2014.

[37] K. A. Moses, Z. Zhao, Y. Bi et al., "The impact of sociodemographic factors and PSA screening among low-income Black and White men: data from the Southern Community Cohort Study," Prostate Cancer and Prostatic Diseases, vol. 20, no. 4, pp. 424-429, 2017.

[38] W. Gorday, H. Sadrzadeh, L. De Koning, and C. Naugler, "Association of sociodemographic factors and prostate-specific antigen (PSA) testing," Clinical Biochemistry, vol. 47, no. 16-17, pp. 164-169, 2014.

[39] N. N. Sanford, D. J. Sher, S. Butler et al., "Cancer screening patterns among current, former, and never smokers in the United States, 2010-2015," JAMA Network Open, vol. 2, no. 5, Article ID e193759, 2019.

[40] G. Carreras, L. Iannucci, G. Costa, E. Chellini, and G. Gorini, "Are smokers less likely to seek preventive healthcare measures in Italy?" European Journal of Cancer Prevention, vol. 27, no. 5, pp. 507-513, 2018.

[41] D. Wilson, B. Smith, I. Speizer et al., "Differences in food intake and exercise by smoking status in adolescents," Preventive Medicine, vol. 40, no. 6, pp. 872-879, 2005.

[42] C. J. Berg, J. E. Painter, J. M. Sales et al., "Smoking as a risk factor for STI diagnosis among african American females," American Journal of Health Behavior, vol. 36, no. 4, pp. 505-512, 2012.

[43] K. R. Fonaine, M. Heo, and D. B. Allison, "Obesity and prostate cancer screening in the USA," Public Health, vol. 119, pp. 694-698, 2005.

[44] R. M. Merrill and R. Fowers, "To what extent does sex, age and BMI impact medical and pharmacy costs? A retrospective cohort study involving employees in a large school district in the USA," BMJ Open, vol. 9, no. 5, Article ID e024078, 2019.

[45] J. S. Ross, E. H. Bradley, and S. H. Busch, "Use of health care services by lower-income and higher-income uninsured adults," JAMA, vol. 295, pp. 2027-2036, 2009. 